\title{
Soya oil-based shampoo superior to $0.5 \%$ permethrin lotion for head louse infestation
}

\author{
This article was published in the following Dove Press journal: \\ Medical Devices: Evidence and Research \\ 10 March 20II \\ Number of times this article has been viewed
}

\author{
Ian F Burgess' \\ Katrina Kay ${ }^{1,2}$ \\ Nazma A Burgess' \\ Elizabeth R Brunton' \\ 'Medical Entomology Centre, Insect \\ Research and Development Limited, \\ Cambridge, ${ }^{2}$ Leeds Primary Care \\ Trust, Leeds, UK
}

Background: This was a randomized, assessor-blind, controlled comparison of a soya oil-based medical device shampoo with a medicinal permethrin lotion in an alcohol vehicle for treatment of head louse infestation to generate data suitable for a regulatory submission to achieve reimbursable status for the shampoo product.

Methods: We treated 91 children and adults, divided between two sites, on two occasions 9 days apart. Participants washed their hair and towel-dried it before treatment. The shampoo was used twice for 30 minutes each time. The lotion was used for 30 minutes followed by rinsing. Assessments were made by dry detection combing on days 2, 9, 11, and 14 after the first treatment. According to present knowledge, this combing technique does not influence the overall head louse populations or outcome of treatment.

Results: The soya oil shampoo was significantly $(P<0.01)$ more effective than the lotion for both intention to treat ( $62.2 \%$ versus $34.8 \%$ successful treatment) and per-protocol $(74.3 \%$ versus $36.8 \%$ success) groups. Post-treatment assessments showed the necessity for repeat treatment, but that a 9-day interval was too long because if eggs hatched after the first treatment, the lice could grow old enough to lay eggs before the second treatment.

Conclusion: The soya oil-based shampoo was more effective than the permethrin lotion, more cosmetically acceptable, and less irritant.

Keywords: pediculosis, medical device, medicinal product, insecticide, vegetable oil

\section{Introduction}

Medical devices, which have a physical mode of action, are widely employed to control head louse infestation in Europe and, more recently, in Australia. However, in most countries, competent authorities and national prescribing schemes still generally favor insecticide-based medicinal products over medical devices for patient reimbursement schemes. This is despite the fact that the efficacy of many of the insecticide products is now reduced because the lice have acquired resistance to the active chemicals. ${ }^{1-4}$

The efficacy of several Class I medical devices compared with insecticides has already been demonstrated in randomized, controlled, comparative studies. ${ }^{5-8}$ Nevertheless, most competent authorities have been reluctant to include devices in their prescribing formularies, so that parents using these products to eliminate infestation from their children can obtain reimbursement of costs. Therefore, many general health practitioners continue to prescribe less efficient and potentially more toxic insecticide-based preparations. This has only served to increase levels of insecticide resistance in countries such as the UK and the Irish Republic.
Correspondence: lan F Burgess Insect Research and Development Limited, 6 Quy Court, Colliers Lane, Stow-cum-Quy, Cambridge,

CB25 9AU, UK

Tel +44 I 223810070

Fax +441223810078

Email ian@insectresearch.com 
The Class I medical device investigated in this study has been available for use in Germany since 2001, and its efficacy has previously been evaluated in preliminary uncontrolled tests. The aim of this study was to demonstrate that the soya oil-based shampoo is effective when used in a community setting. The manufacturers also wished to generate evidence for efficacy, in comparison with the leading medicinal product marketed in Germany, so they could make an application to the authorities for approval for inclusion on the reimbursable listing.

\section{Methods and materials Objective}

We designed the study so we could analyze the results in two stages. The first stage would allow us to show that the soya oil-based shampoo demonstrated noninferiority to $0.43 \% \mathrm{w} / \mathrm{v}$ permethrin lotion. The design was sufficiently powered that a second-stage analysis could then be performed to detect if either product showed superior activity. We assumed that all participants were independent.

\section{Participants}

We recruited participants into the study in essentially the same way as for previous studies, by advertising on local radio, in newspapers, and by contact with previous study participants. Potential participants were supplied with an information booklet prior to recruitment, and an enrolment visit was arranged a minimum of 24 hours later.

All prospective participants signed consent forms before being screened for the presence of at least five live head lice, found within a maximum screening time of 5 minutes, by using a plastic detection comb (KSL Consulting, Helsinge, Denmark, Figure 1). Each infested household member could

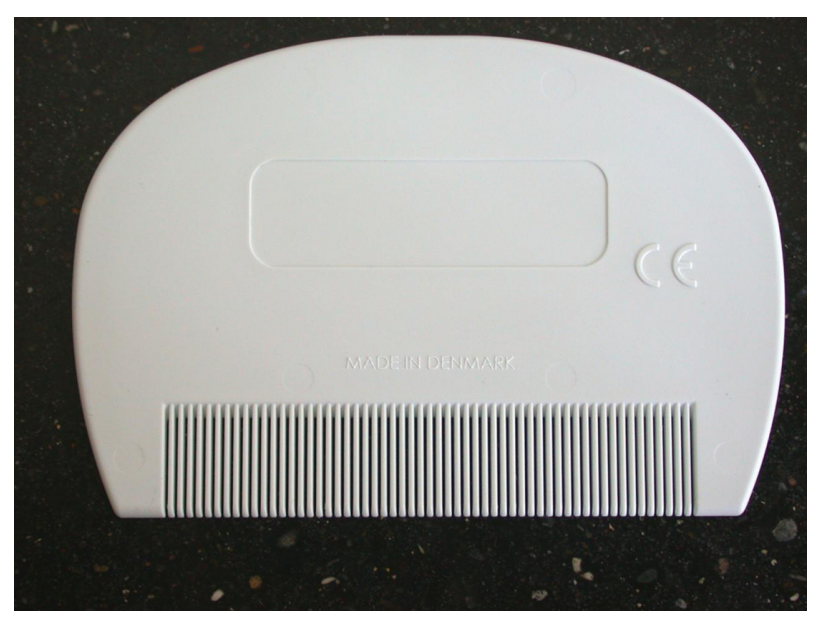

Figure I The 'PDC' head louse detection comb. be enrolled if: older than 2 years; they had not been treated with head louse products during the previous 2 weeks; and that had not been treated with trimethoprim-sulfamethoxazole or permanent waves or hair colors during the previous 4 weeks. We asked all prospective participants to confirm that they were unaware of allergy or sensitivity to pyrethroid insecticides, soya, nuts, or any other ingredient of the test products. Adult females also confirmed that they were not breast feeding or pregnant, and using adequate contraception. We excluded people with asthma or similar respiratory conditions and those with long-term scalp conditions other than pediculosis, earlier participants in this trial, and anyone who had participated in another clinical study within one month before entry.

We collected baseline demographic data on gender, age, hair characteristics, and previous pediculicide use. All treatments and assessment visits were domiciliary. No payment was offered for participation. Any ineligible people with lice were offered a standard of care treatment ( $4 \%$ dimeticone liquid gel) to prevent reinfestation of study participants.

\section{Ethics}

Ethical approval for the study was granted by the Oxfordshire Research Ethics Committee A. The study was conducted in conformity with the principles of the Declaration of Helsinki, of the European Union Directive 2001/20/EC, and the ICH Topic E11 guideline. All participants stated before giving consent that they had read the participation information booklet and understood the purpose and requirements of the study. Parents or guardians gave written consent for children younger than 16 years. Children also provided written or verbal assent, according to age, witnessed by the parent or guardian. The study was registered with the Current Controlled Trials database (ISRCTN48669688).

\section{Sample size and randomization}

A total sample size of 96 participants (48 in each of the treatment groups) was considered sufficient to detect a difference of $35 \%$ between groups for success rate at 14 days, with $90 \%$ power and $95 \%$ confidence intervals (CI). This $35 \%$ difference represented the difference between a $35 \%$ success rate in one product group and a $70 \%$ rate in the other group. The actual sample size required was 45 per group, so a planned recruitment of 48 per group made allowance for dropout.

Treatment allocation was derived from an online computer-generated list. ${ }^{9}$ Allocation at the point of delivery was made from instruction sheets enclosed in opaque, sealed, sequentially numbered envelopes distributed to investigators in balanced blocks of eight. A copy of the listing was prepared 
in case an emergency code break was required. Participants were allocated the next numbered envelope available to the investigator. Randomization was by individual, so different family members could receive different treatments.

\section{Treatment}

Not only were the products physically different, the method of application was completely different also, so blinding at delivery was impossible. This study was single-blinded, with post-treatment assessments performed by different investigators, unaware of which treatment products had been used (assessor-blinded). In order to ensure a correct treatment, application was made in conformity with the instructions for use supplied with each product.

The soya oil shampoo (Mosquito ${ }^{\circledR}$ LäuseShampoo; Wepa Apothekenbedarf GmbH and Co KG, Hillscheid, Germany) was supplied in $100 \mathrm{~mL}$ bottles. It was first used to wash the hair normally, followed by towel drying. A greater quantity of shampoo (about three times the quantity required to wash the hair) was then applied and massaged to produce a stable foam, with more added if the foam broke too quickly. We left the shampoo on the hair for 30 minutes, covered with a shower cap to prevent drips. At the end of this time it was rinsed off, towel-dried, and reapplied in the same manner. After a further 30 minutes, the shampoo was rinsed out.

We applied the permethrin lotion (InfectoPedicul ${ }^{\circledR}$ lotion; Infectopharm Arzneimittel und Consilium $\mathrm{GmbH}$, Heppenheim, Germany), which was supplied in $150 \mathrm{~mL}$ bottles, after washing the hair with a nonmedicated shampoo followed by towel-drying. The lotion was applied a few drops at a time and massaged through the hair until the hair was soaked to the point of runoff. It was left in place for 30 minutes and then rinsed off with water. Parents or guardians performed the rinsing, in some cases while the investigator was still present. We asked those people treated with permethrin lotion not to shampoo their hair for 3 days.

For both products, a second treatment was applied 9 days later. Participants were reminded not to use nit combs or other pediculicide preparations during the course of the study.

\section{Statistics and outcome measures}

The primary outcome measure for the study was elimination of lice after two applications of product. Assessments were made by dry detection combing on day 2 , on day 9 immediately before the second treatment, then day 11, and a more thorough examination was performed on day 14. Any lice found were collected in the case record and examined by microscope to determine the development stage. If no lice were found on days 11 and 14, after the second application of treatment, it was considered a success.

Statistical analyses were performed blind of treatment allocation. Fisher's exact tests were used for presence/ absence variables. Differences in success rates were measured by the $95 \%$ CI calculated using a normal approximation of the binomial distribution. Quantitative variables were compared using an unpaired $t$-test or the Mann-Whitney $U$ test. We conducted analyses using Oxstat II (version 1.1; Oxstat Ltd, London, UK), Epi Info (version 6; Centers for Disease Control and Prevention, Atlanta, GA), and purpose-built calculators for normal approximation and Kruskal-Wallis/ Mann-Whitney tests.

\section{Results}

\section{Participants}

The study was a two-center investigation, with sites about 150 miles apart in Cambridge and Leeds, UK. Between June 21, 2010 and December 31, 2010, consent was obtained from 134 people to be screened for head lice, and 91 participants (59 Cambridge, 32 Leeds) in 48 households were enrolled in the study. A further 121 household members either declined screening, were ineligible, or were unavailable. Enrolled people comprised 78 children and 13 adults aged 2-47 (median 10) years. Most demographic characteristics of the study population showed no significant differences when compared with populations enrolled in previous studies. ${ }^{5,6,8}$ However, the proportion of participants with heavier infestations at enrolment (ie, more than one louse found with the first stroke of a detection comb) was significantly $(P<0.001)$ higher in Leeds than in Cambridge, although this did not appear to influence the outcome of treatment.

More than one family member in 24 households participated, 14 families having two participants in the study, seven families had three participants, and three families had four. The most common household sizes were five (15 houses), three (10 houses), and four (10 houses). It was planned that equal numbers of people would receive each treatment (48 per group), with a final enrolment of 45 receiving shampoo and 46 receiving lotion in the time allocated by the sponsor. Eighty-six participants were assessed on day 14, and were considered to have completed the study (Figure 2). Overall, there were 18 instances of noncompliance. One child refused a second application of permethrin lotion and two refused a second application of shampoo, because the treatments were too irritating. Six participants dropped out, and five parents combed lice from their child's hair between treatments (two on permethrin lotion and three on 


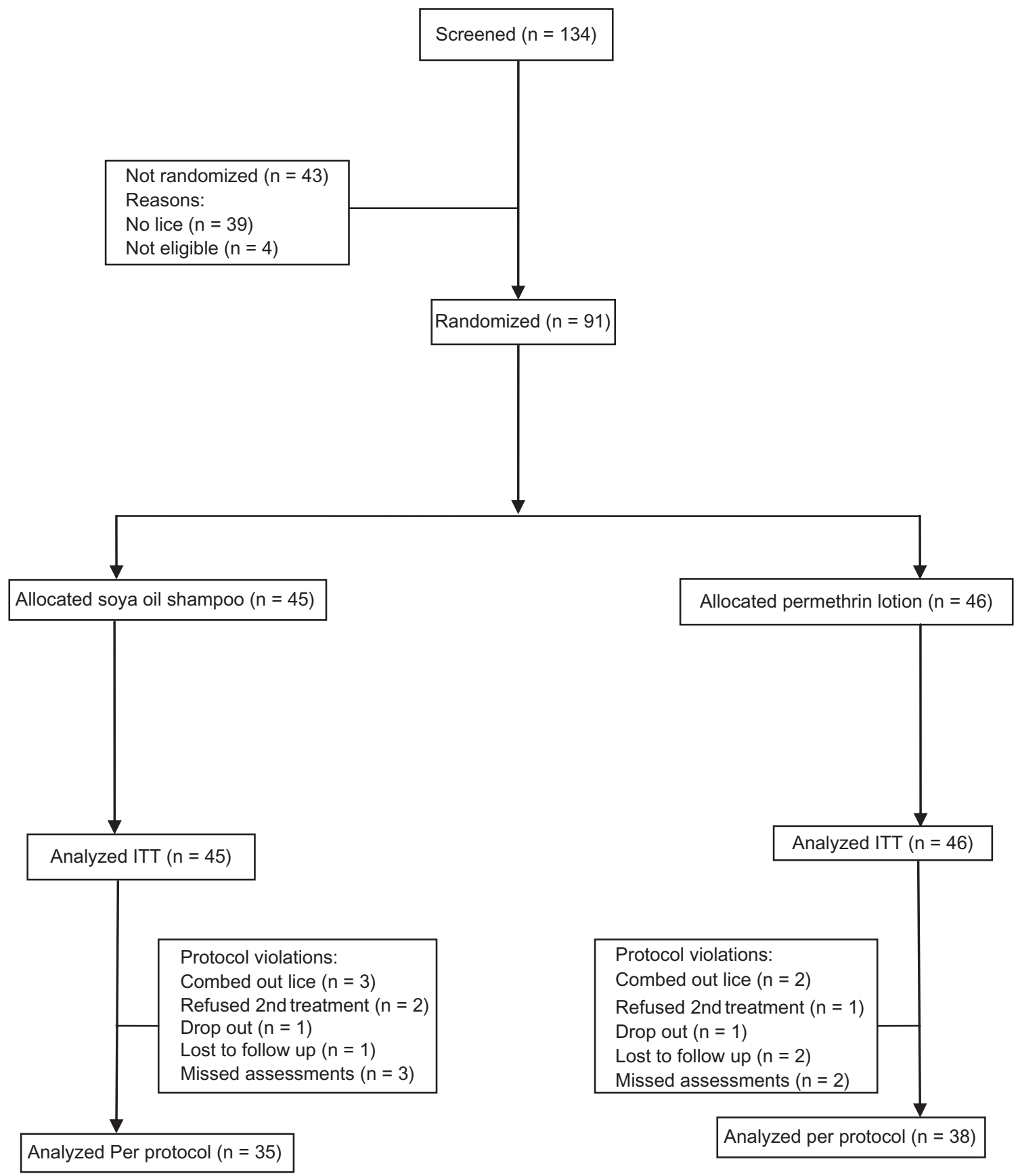

Figure 2 Flowchart of participants through the study.

soya oil shampoo), and the remainder missed one or more other assessments. Data from these participants were included in the intention to treat analyses. All other participants had complete data sets, with two treatments nine days apart, and follow-up checks on days 2, 9, 11, and 14 .

The main endpoint analysis was the comparison of rate of cure for the 91 participants in the intention to treat population. According to this criterion, success was achieved by $16 / 46$ (34.8\%) of the participants in the permethrin lotion group and by $28 / 45(62.2 \%)$ of the participants in the soya oil shampoo group. The difference in rate of success between the two treatments was estimated as $27.4 \%$ (95\% CI: $7 \%$, $48 \%$ ) which meant that the soya oil shampoo was significantly $(P<0.01)$ more effective than permethrin lotion in the population tested.

Elimination of protocol violators from the analysis gave per-protocol success rates of $36.8 \%$ for the permethrin lotion group and $74.3 \%$ for the soya oil shampoo group, a difference of $37.5 \%$ (95\% CI: $15 \%, 60 \%$ ), which was also significant $(P<0.001)$. 
Comparison of outcomes for the two sites found a difference in success rates for both intention to treat and perprotocol analyses. In Cambridge, lice were eliminated in $21 / 29(72.4 \%)$ of the intention to treat population treated with shampoo compared with $7 / 16(43.8 \%)$ in Leeds, and 11/30 (36.7\%) had no lice after treatment with permethrin lotion in Cambridge compared with 5/16 (31.3\%) in Leeds. Similar differences in outcomes were found in the per-protocol population, with success in 21/26 (80.8\%) for shampoo and 10/25 (40.0\%) for lotion in Cambridge compared with 5/9 (55.6\%) success for shampoo and 4/13 (30.8\%) for lotion in Leeds.

Analysis of lice collected post-treatment showed that there were significantly more louse nymphs present at days 9 and 11 following the lotion treatment than after using shampoo (Table 1). We found that 10 participants treated with soya oil shampoo had no lice at any checkup after the first application of product compared with five people treated with permethrin lotion. However, most participants in both groups, irrespective of whether lice were found at the day 2 assessment, were found to have adult lice present by the time of the day 9 assessment ( 24 shampoo and 25 lotion), all but seven of whom had female lice containing fully developed eggs, demonstrating the absolute necessity of a second application of product.

We weighed the bottles of product before and after use to measure product usage. The mean quantity of shampoo applied $(65.2 \mathrm{~g})$ per treatment was similar to the quantity of lotion $(60.5 \mathrm{~g})$ given.

\section{Adverse events}

There were 41 adverse events in 30 participants. Of these, 13 were simple accidents or childhood infections not related to treatment. All other adverse events were some form of hot, itching, or stinging sensation during one or both treatments ( 12 people [26.6\%] with the shampoo and 11 [23.9\%] with the lotion). Five of the participants experienced two treatment-related adverse events each, two treated with the shampoo and three with the lotion (Table 2). The most severe adverse events were in children treated with the lotion, in which the high concentration of alcohol caused intense stinging of excoriations on the scalp, such that at least three children found it so distressing they tried to wipe the lotion off, and one refused a second treatment. At least two participants found that removing the shower cap supplied with the shampoo reduced irritation during treatment with that product.

\section{Discussion}

In this comparison of a medical device (soya oil-based shampoo) and a medicinal product (permethrin alcoholic lotion), we have found that the physically acting shampoo was significantly more effective than the insecticide.

Table I Comparison of the number of lice at each development stage found at post-treatment assessments

\begin{tabular}{|c|c|c|c|c|}
\hline \multirow[t]{2}{*}{ Assessment day } & \multirow[t]{2}{*}{ Louse life stage } & \multicolumn{2}{|c|}{ Mean number of lice [SD] } & \multirow[t]{2}{*}{$P$ value } \\
\hline & & Shampoo & Lotion & \\
\hline \multirow[t]{5}{*}{ Day 2} & \#I nymph & $0.4[0.7]$ & $1.8[6.4]$ & NS \\
\hline & \#2 nymph & $0.7[1.3]$ & $0.6[1.8]$ & NS \\
\hline & \#3 nymph & $0.6[1.3]$ & $0.5[1.4]$ & NS \\
\hline & Male & $0.6[1.8]$ & $0.8[3.0]$ & NS \\
\hline & Female & $0.4[1.0]$ & I.6 [4.4] & NS \\
\hline \multirow[t]{5}{*}{ Day 9} & \#I nymph & $0.2[0.6]$ & $\mathrm{I} .7[3.7]$ & $<0.001$ \\
\hline & \#2 nymph & $0.2[0.6]$ & $\mathrm{I} .8[2.7]$ & $<0.001$ \\
\hline & \#3 nymph & $0.2[0.5]$ & $2.9[6.0]$ & $<0.001$ \\
\hline & Male & $0.8[1.2]$ & $0.9[1.7]$ & NS \\
\hline & Female & $1.4[2.0]$ & I.3 [I.7] & NS \\
\hline \multirow[t]{5}{*}{ Day II } & \#I nymph & $0.0[0.0]$ & $0.6[2.4]$ & $<0.05$ \\
\hline & \#2 nymph & $0.0[0.0]$ & $0.7[1.7]$ & $<0.01$ \\
\hline & \#3 nymph & $0.1[0.3]$ & $0.6[1.3]$ & $<0.01$ \\
\hline & Male & $0.1[0.3]$ & $0.3[0.7]$ & NS \\
\hline & Female & $0.2[0.5]$ & $0.6[1.0]$ & $<0.01$ \\
\hline \multirow[t]{5}{*}{ Day 14} & \#I nymph & 0.4 [1.9] & $0.9[3.4]$ & NS \\
\hline & \#2 nymph & $0.2[0.9]$ & 0.8 [2.6] & NS \\
\hline & \#3 nymph & $0.1[0.2]$ & 0.8 [3.3] & NS \\
\hline & Male & $0.2[0.6]$ & $0.3[0.7]$ & NS \\
\hline & Female & 0.3 [I.2] & 0.9 [I.4] & $<0.01$ \\
\hline
\end{tabular}

Abbreviations: SD, standard deviation; NS, not significant. 
Table 2 Comparison of adverse events considered related to treatment

\begin{tabular}{|c|c|c|c|}
\hline Participant & Adverse event & Timing & Severity \\
\hline \multicolumn{4}{|c|}{ Soya based shampoo } \\
\hline 010 & Stinging & During treatment & Mild \\
\hline $0 \mid 3$ event $\mid$ & Felt hot on head & During 2nd application of Ist treatment & Mild \\
\hline 013 event 2 & Felt hot on head & During Ist application of 2 nd treatment & Mild \\
\hline 015 & Scalp very itchy & During 2nd treatment & Mild \\
\hline 032 event I & Burning sensation & Ist treatment & Moderate \\
\hline 032 event 2 & Burning sensation & 2nd treatment & Moderate \\
\hline 050 & Intense itching & 15 minutes into treatment & Mild \\
\hline 056 & Intense itching & While treatment on scalp & Mild \\
\hline 058 & Sore burning scalp & During and after treatment & Moderate \\
\hline 060 & Head extremely itchy & During Ist treatment & Mild \\
\hline 061 & Stinging of scalp & Within 3 minutes of application & Mild \\
\hline 068 & Scalp hot and itchy & When shower cap in place & Mild \\
\hline 077 & Scalp very itchy & During treatment & Mild \\
\hline 086 & Stinging & During treatment & \\
\hline \multicolumn{4}{|c|}{$0.5 \%$ permethrin lotion } \\
\hline 011 & Stinging of scalp & During application & Mild \\
\hline 012 & Stinging of scalp & During application & Mild \\
\hline 055 & Severe stinging of scalp & As product applied & Moderate \\
\hline 064 event I & Severe stinging of scalp & During Ist treatment & Moderate \\
\hline 064 event 2 & Intense stinging of scalp & During 2nd treatment & Severe \\
\hline 068 event I & Stinging of scalp & During Ist treatment & Mild \\
\hline 068 event 2 & Stinging of scalp & During 2 nd treatment & Mild \\
\hline 066 & Intense stinging of scalp & During application & Severe \\
\hline 067 event I & Stinging of scalp & When product applied Ist treatment & Mild \\
\hline 067 event 2 & Stinging of scalp & When product applied 2nd treatment & Mild \\
\hline 074 & Stinging of scalp & During Ist treatment & Mild \\
\hline 080 & Intense itching & After product washed off & Mild \\
\hline 081 & Scalp felt hot & 10 minutes into treatment & Mild \\
\hline 096 & Burning/stinging & During application & Mild \\
\hline
\end{tabular}

Indications from other studies conducted in the UK suggest that lack of efficacy for the lotion is because permethrin currently has limited effectiveness due to physiological degradation mechanisms rather than gene mutations such as $k d r$ (knockdown resistance). ${ }^{2,8,10}$

In this study, we followed the instructions for use of the products as supplied by the manufacturers, with reapplication after nine days (the product leaflets ${ }^{11,12}$ suggest reapplication after 8-10 days). Evidence obtained from lice collected during the assessments not only suggested that neither product exerts a complete ovicidal effect, but also that the interval between treatments is too long, because nymphs emerging after the first treatment had, in many cases, developed into fully matured adults by the time of the second treatment. Adult female lice collected on day 9 mostly contained fully developed eggs and, in one case, eggs appeared to have already been laid by these lice, because new first-stage nymphs were found at the day 14 assessment. There are no published data on the time required for head louse eggs to hatch on the head (all published studies have been either of body louse eggs or of head louse eggs maintained in the laboratory or in containers on other parts of the body). There are also no clear data for how long lice take to mature. What studies exist suggest is that maturation of lice can occur in around 8 days. ${ }^{13}$ Therefore, it has been agreed by several investigators around the world that a 7-day interval is more likely to provide the best outcome (Barker et al, unpublished data).

We found a difference in outcome between the two study sites. Any difference in efficacy of permethrin lotion could be attributed to differences in resistance of lice in each locality, but this could not explain differences in the efficacy of the soya oil shampoo. We can only suggest that any difference may have been due to individual variation in application technique within the instructions by different investigators. One factor we found critical was the quantity of water remaining on the hair and, in Cambridge, this was reduced to an absolute minimum by rigorous towel drying prior to the therapeutic application of product by one of us (IFB). Other investigators may have been less rigorous, which could have resulted in greater dilution of the product.

Physically acting medical device products have increased in number, availability, and variety of dosage form. 
All essentially act either by occlusion of the louse respiratory tract or by disruption of the surface lipids of the insects. ${ }^{14,15}$ As a result, this type of product is popular with consumers in most countries because they do not contain conventional insecticides, and mostly do not contain harsh solvents, such as alcohol. The disadvantages of alcohol were clear in this study because a high proportion (31\%) of those treated with the permethrin lotion reported mild to intense stinging when the fluid was applied to louse bites and excoriations on the scalp. The distress caused to younger children was severe. However, even the shampoo was not without some side effects resulting from relatively prolonged contact with surfactant components. Such effects were exacerbated by use of the shower cap, which probably does not help the therapeutic activity in any way. Nevertheless, in comparison with the insecticide-based lotion, the soya oil shampoo was not only more acceptable than the lotion but was also significantly more effective, with a success level similar to several other widely used medical devices..$^{5-8}$

The mosquito LäuseShampoo contains soya oil, which is made up from several medium chain, mostly unsaturated, lipids (C16:0, C18:0, C18:1, C18:2, C18:3). Soya oil has a similar profile to other fixed vegetable oils, such as neem oil and olive oil, that are reputed to have a pediculicidal occluding effect. ${ }^{16}$ The mechanism of action of such oils on head lice is unknown, although it has been speculated in the popular press that they have some form of asphyxiation effect. Because insects have a waxy protective layer on their cuticle that contains lipids of similar carbon chain lengths, ${ }^{17}$ what is more probable is that the lipids in the shampoo, combined with surfactant, cause disruption to the surface lipids of the lice, leading to water loss and dehydration.

\section{Conclusion}

In this randomized, controlled, assessor-blinded, clinical investigation we have shown that a soya oil-based shampoo (Mosquito LäuseShampoo) was significantly more effective than a permethrin lotion with an alcohol vehicle. The product was better tolerated and more cosmetically acceptable. However, the recommended retreatment time should be adjusted from 8-10 days to 7 days in order to avoid the risk that louse nymphs hatching from eggs surviving the first treatment may develop into adulthood and lay viable eggs themselves before the second application of product.

\section{Acknowledgments}

Our thanks also go to Dietmar Mühlhofer of LHS Institut für Hygieneforschung und Schädlingsbekämpfung in Labor und Praxis, Wiener Neustadt, Austria, and to Dr Wilfried Dierkes of Diapharm Clinical Management GmbH, Oldenburg, Germany, for support and advice. Investigator members of the study team not listed as authors were Audrey Pepperman and Benedict Hall, Cambridge, and Elizabeth Walters, Christina Bulley, Susan Hunter, and Dr Mike Gent, Leeds. Thanks also to Laura Esteras, Janet Selby, and José Vicente-Garcia of Harrison Clinical Research Ltd, Ely, UK, for monitoring of study documents. Dr Paul Silverston was the study consultant physician who reviewed all adverse events.

\section{Disclosure}

This study was sponsored and financed by Wepa Apothekenbedarf $\mathrm{GmbH}$ and Co KG, Hillscheid, Germany. IFB is a consultant to several major and minor manufacturers of pediculicides, medical devices, and combs for use against head lice and their eggs. The other authors report no conflicts of interest in this work.

\section{References}

1. Rupeš V, Moravec J, Chmela J, Ledvinka J, Zelenková J. A resistance of head lice (Pediculus capitis) to permethrin in Czech Republic. Cent Eur J Public Health. 1995;3:30-32.

2. Downs AMR, Stafford KA, Harvey I, Coles GC. Evidence for double resistance to permethrin and malathion in head lice. Br J Dermatol. 1999;141:508-511.

3. Thomas DR, McCarroll L, Roberts R, et al. Surveillance of insecticide resistance in head lice using biochemical and molecular methods. Arch Dis Child. 2006;91:777-778.

4. Kristensen M, Knorr M, Rasmussen A-M, Jespersen JB. Survey of permethrin and malathion resistance in human head lice populations from Denmark. J Med Entomol. 2006;43:533-538.

5. Burgess IF, Lee PN, Matlock G. Randomised, controlled, assessor blind trial comparing $4 \%$ dimeticone lotion with $0.5 \%$ malathion liquid for head louse infestation. PLoS One. 2007;2:e1127.

6. Burgess IF, Lee PN, Brown CM. Randomised, controlled, parallel group clinical trials to evaluate the efficacy of isopropyl myristate/ cyclomethicone solution against head lice. Pharm J. 2008;280: 371-375.

7. Heukelbach J, Pilger D, Oliveira FA, Khakban A, Ariza L, Feldmeier H A highly efficacious pediculicide based on dimeticone: Randomized observer blinded comparative trial. BMC Infect Dis. 2008;8:115.

8. Burgess IF, Brunton ER, Burgess NA. Clinical trial showing superiority of a coconut and anise spray over permethrin $0.43 \%$ lotion for head louse infestation, ISRCTN964697. Eur J Pediatr. 2010;169:55-62.

9. Randomization sequence generator [online resource]. Available from: http://www.randomization.com, seed 6118. Accessed May 26, 2010.

10. Burgess IF, Brown CM. Management of insecticide resistance in head lice, Pediculus capitis (Anoplura: Pediculidae). In: Robinson WH, Rettich F, Rambo GW, editors. Proceedings of the 3rd International Conference on Control of Urban Pests, Prague, Czech Republic, July 19-22, 1999.

11. Mosquito ${ }^{\circledR}$ LäuseShampoo (glycine soya shampoo) [Package insert]. Hillscheid, Germany: Wepa Apothekenbedarf; 2004.

12. InfectoPedicul $(0.43 \% \mathrm{w} / \mathrm{v}$ permethrin lotion) [Package insert] Heppenheim, Germany: Infectopharm Arzneimittel; 2006.

13. Takano-Lee M, Yoon KS, Edman JD, Mullens BA, Clark JM. In vivo and in vitro rearing of Pediculus humanus capitis (Anoplura: Pediculidae). J Med Entomol. 2003;40:628-635. 
14. Burgess IF. The mode of action of dimeticone $4 \%$ lotion against head lice, Pediculus capitis. BMC Pharmacol. 2009;9:3.

15. Mougabure Cueto G, Gonzalez Audino P, Vassena CV, Picollo MI, Zerba EN. Toxic effect of aliphatic alcohols against susceptible and permethrin-resistant Pediculus humanus capitis (Anoplura: Pediculidae). J Med Entomol. 2002;39:457-460.
16. Carpinella MC, Miranda M, Almirón WR, Ferrayoli CG, Almeida FL, Palacios SM. In vitro pediculicidal and ovicidal activity of an extract and oil from fruits of Melia azedarach L. J Am Acad Dermatol. 2007;56: 250-256.

17. Jackson LL, Baker GL. Cuticular lipids of insects. Lipids. 1969;5: 239-246.

\section{Publish your work in this journal}

Medical Devices: Evidence and Research is an international, peerreviewed, open access journal that focuses on the evidence, technology, research, and expert opinion supporting the use and application of medical devices in the diagnosis, treatment and management of clinical conditions and physiological processes. The identification of novel devices and optimal use of existing devices which will lead to improved clinical outcomes and more effective patient management and safety is a key feature. The manuscript management system is completely online and includes a quick and fair peer-review system. Visit http://www. dovepress.com/testimonials.php to read real quotes from authors.

Submit your manuscript here: http://www.dovepress.com/medical-devices-evidence-and-research-journal 